\title{
Bio-Chemo-Opto-Mechanical (BioCOM) Sensors for Real-Time Characterization for D\&D Applications
}

\section{Research Objective}

Basic and applied research will be conducted to develop simple to use chemical and biological sensor chips utilizing bio-chemo-mechanics for real-time, in-situ, detection of technetium, mercury, uranium, and beryllium for deactivation and decommissioning applications. The bio-chemo-opto-mechanical (Bio-COM) chip involves properly fashioned arrays of micromachined silicon cantilevers containing embedded deformable diffraction gratings functionalized with chemically selective coatings. Adsorption of specific molecules on the cantilever array leads to bending, which changes the diffraction of light from the array. The bio-chemo-opto-mechanical (BioCOM) chips will be designed to contain an array of pixels, with each pixel containing an array of microcantilever springs in which one surface is derivatized with either an antibody coating or a self-assembled monolayer (SAM) coating for detecting $\mathrm{Hg}, \mathrm{Be}$, $\mathrm{U}(\mathrm{VI})$, or $\mathrm{TcO}_{4}$. The BioCOM sensor platform also offers the advantage of simultaneous measurement of many analytes using a single chip. The readout mechanism can be a reflected laser beam, producing a diffraction pattern, or in an ideal case the diffraction of daylight resulting in a change of color. In the latter case the proposed sensors would not require any external power, external or on-board electronics, or fluorescent dyes and associated optics, which will keep its fabrication and operation costs low while making it simple to use for real-time environmental monitoring.

\section{Research Progress and Implications}

This report summarizes the work in the first year (June, 2002) of a three year project. The three basic goals of this program are the basic research to design and develop coatings to make selective sensors, the design and fabrication of the test chip and the optical detection system, and the design and development and field test of a prototype sensor. The latter general goal will be carried out in the third year, and any discussion of a field test is premature at this juncture. However good progress has been made toward advancement of the first two goals.

Chip Design and Microfabrication and Optical Detection System Design. Professor Arun Majumdar's group at UC Berkeley has been involved in the design, fabrication, and operation of a novel generation of non-electronic micromechanical chips containing arrays of microcantilever beams. The deformable diffraction grating chips are fabricated by a surface micromachining process, and as shown in the figure the BioCOM chips are an interdigitated array. The interdigitated fingers of all the cantilevers on the chip collectively reflect the incident energy of monochromatic laser light into well-defined directions that correspond to the zeroth, first, second, and $\mathrm{n}^{\text {th }}$ mode of diffraction. Each of these modes contains sufficient information to reproduce the image that is made of the deflections of all the cantilever beams. This technology 


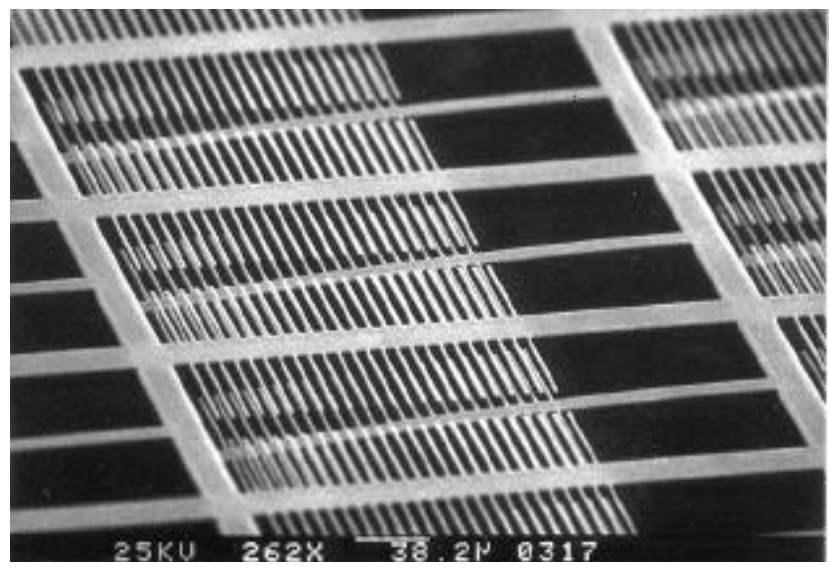

will be used as one of the approaches for the BioCOM chip to produce a color signal from incident white light. Work is in progress to develop a method of electronically recording the diffraction patterns as a function of time so that time dependent sorption studies can be conducted.

A higher density of "fingers" will be required to observe color changes as the interdigitated cantilever bends. Work is in progress at the University of California-Berkeley to prepare chips with this property.

Design and Development of Selective Coatings. Work is in progress to adapt the surface chemistry to attach metal specific antibodies to the surface of the cantilever chips. Initial work is being conducted using AFM cantilevers while we await the delivery of interdigitated cantilevers from the UC-Berkeley. The following is the strategy for immobilizing antibodies onto the cantilever surface. (i) covalently functionalize the cantilever surface with a linker/modifier molecule; and (ii) covalently link antibodies or ligands to the modifier. Two different linkers are under investigation. Gold coated cantilevers will be functionalized with heterobifunctional crosslinkers [e.g. DSP (dithiobis(succinimidylproprionate))]containing thiol groups (reactive to gold) and N-hydroxy succinimide esters (reactive to antibody primary amine groups). The carboxylic acid moieties on the $\mathrm{C}$-terminus and glutamic acid and aspartic acid residues can be coupled to amine functionalized cantilever surfaces via EDC-mediated amide bond formation. EDC [1-ethyl-3-(3-dimethylaminopropyl)carbodiimide] reacts with carboxylic acid groups to form a highly reactive isourea derivative that reacts with primary amines to form a very stable peptide bond. Furthermore, work is in progress to attach pertechnetate and uranylcarbonate selective ligands to the surface for specificity. Quarternary ammonium salts, tethered to the gold coating with an thiol linking group are being tested for the $\mathrm{TcO}_{4}{ }^{-}$and $\mathrm{UO}_{2}\left(\mathrm{CO}_{3}\right)_{3}{ }^{4-}$ species.

\section{Planned Activities}

In the remainder of this fiscal year, work will continue on the development of compound specific coatings as well as on an electronic readout In the socnd year of the project we will focus our efforts on the further development of techniques for applying selective coatings on the BioCOM chip, and we will attempt to demonstrate the selectivity of BioCOM chips for $\mathrm{Hg}^{2+}$, $\mathrm{Be}(\mathrm{II}), \mathrm{TcO}_{4}{ }^{-}$, and $\mathrm{U}(\mathrm{VI})$ dissolved in solution.

\section{Information Access}

T. Thundat, E. Finot, Z. Hu, R.H. Ritchie, G. Wu, and A. Majumdar, "Chemical Sensing in Fourier Space," Appl. Phys. Lett. 77, 24, 4061-63 (2000). 